\title{
Growth Phase-Differential Quorum Sensing Regulation of Anthranilate Metabolism in Pseudomonas aeruginosa
}

\author{
Yusang Choi ${ }^{1,2}$, Ha-Young Park, ${ }^{1,2}$, Seong Joon Park ${ }^{1}$, Su-Jin Park ${ }^{1}$, Soo-Kyoung Kim ${ }^{1}$, Changwan Ha ${ }^{1}$, \\ Su-Jin $\mathrm{Im}^{1}$, and Joon-Hee Lee ${ }^{1, *}$
}

\begin{abstract}
Pseudomonas quinolone signal (PQS) plays a role in the regulation of virulence genes and it is intertwined in the las/rhl quorum sensing (QS) circuits of Pseudomonas aeruginosa. PQS is synthesized from anthranilate by pqsA-D and pqsH whose expression is influenced by the las/rhl systems. Since anthranilate can be degraded by functions of ant $A B C$ and catBCA, PQS synthesis might be regulated by the balance between the expression of the pqsA-D/phnAB, pqsH, antABC, and catBCA gene loci. ant $A$ and cat $A$ are repressed by LasR during log phase and activated by RhIR in late stationary phase, whereas pqsA-E/phnAB is activated by LasR in log phase and repressed by RhIR. QscR represses both but each repression occurs in a different growth phase. This growth phasedifferential regulation appears to be accomplished by the antagonistic interplay of LasR, RhIR, and QscR, mediated by two intermediate regulators, AntR and PqsR, and their cofactors, anthranilate and $P Q S$, where the expressions of ant $R$ and $p q s R$ and the production of anthranilate and $P Q S$ are growth phase-differentially regulated by $Q S$ systems. Especially, the anthranilate level increases in an RhIRdependent manner at late stationary phase. From these results, we suggest that RhIR and LasR regulate the anthranilate metabolism in a mutually antagonistic and growth phase-differential manner by affecting both the expressions and activities of AntR and PqsR, and that QscR also phase-differentially represses both LasR and RhIR functions in this regulation.
\end{abstract}

\section{INTRODUCTION}

Pseudomonas aeruginosa is an opportunistic pathogen that causes serious infections in plants, animals, and humans. This versatile and ubiquitous organism is a major cause of nosocomial infections and is responsible for chronic lung infections in immunocompromised individuals and those suffering from cystic fibrosis (Van Delden and Iglewski, 1998). As in many bacteria, the expression of numerous virulence factors in this bacte- rium is controlled by small diffusible signaling molecules in a cell density-dependent manner known as 'quorum sensing' (QS) (Fuqua et al., 2001; Kim et al., 2009). In P. aeruginosa, QS relies on the activation of specific transcriptional regulators (LasR and RhIR) by corresponding signals synthesized by their cognate acyl HSL synthases (Lasl and Rhll). The signals produced by Lasl and Rhll are $\mathrm{N}$-(3-oxododecanoyl)-L-homoserine lactone (3OC12) and $N$-butanoyl-L-homoserine lactone (C4), respectively. $P$. aeruginosa has a third LasR-RhIR homolog, QscR which was known as an orphan receptor (Chugani et al., 2001), but it was recently reported that QscR shares 3OC12 as its cognate signal with LasR (Lee et al., 2006). These three QS regulators and two signal synthases comprise a hierarchical cascade, where las regulates $r \mathrm{hl}$ and $q s c R$, which co-ordinate the expression of numerous genes (more than $6 \%$ of the $P$. aeruginosa genome), many of which encode virulence and survival factors (Lequette et al., 2006; Schuster and Greenberg, 2006; Schuster et al., 2003; Venturi, 2006).

In addition to acyl-HSLs, another type of signal molecule, Pseudomonas quinolone signal (PQS; 2-heptyl-3-hydroxy-4quinolone), is produced by $P$. aeruginosa and plays a role in the regulation of virulence genes (Pesci et al., 1999). PQS signaling seems intricately intertwined in the QS circuits and is required for the expression of some RhIR-dependent genes at the onset of the stationary phase (Diggle et al., 2003; McKnight et al., 2000; Pesci et al., 1999). While the PQS functions are not fully understood yet, the metabolic pathway and genes for $P Q S$ biosynthesis have been partly elucidated. The precursor is anthranilate (Calfee et al., 2001), from which PQS is synthesized by the products of the pqsABCD and phnAB gene cluster and pqsH (Deziel et al., 2004; Gallagher et al., 2002) (Fig. 1). Because anthranilate is also a precursor of tryptophan biosynthesis (Calfee et al., 2001; Essar et al., 1990; Oglesby et al., 2008) and can be degraded into TCA cycle intermediates by the functions of antABC and catA (encoding anthranilate 1,2dioxygenase complex and catechol 1,2-dioxygenase, respectively) (Oglesby et al., 2008), anthranilate is an important intermediate at the metabolic branch point during the PQS biosynthesis (Fig. 1).

\footnotetext{
${ }^{1}$ Department of Pharmacy, College of Pharmacy, Pusan National University, Busan 609-735, Korea, ${ }^{2}$ These authors contributed equally to this work *Correspondence: joonhee@pusan.ac.kr
} 
Previous transcriptome analysis of QS signaling showed that ant $A B C$ in anthranilate degradation is uniquely regulated by the QS system in a very complicated manner; its expression was induced at late stationary phase in signal mutant (las/, rhlI), repressed by the addition of $30 \mathrm{OC} 12$ and restored again by the co-addition of C4 with 30C12 (Schuster et al., 2003). A recent real-time PCR analysis showed RhIR-dependent activation and LasR-dependent repression of antA (Oglesby et al., 2008). These results indicated that the anthranilate-degrading function of the ant $A B C$ operon might be antagonistically regulated by the QS regulators. Also, the expression of the pqsABCDE/ phnAB operon for the PQS synthesis is also regulated by QS systems where they are activated by las system and repressed by $r h$ l system (McGrath et al., 2004; Wade et al., 2005). These results further imply that $P Q S$ biosynthesis could be finely tuned by the QS systems at the metabolic branch point, anthranilate. Interestingly, these complicated regulations appear to occur with different timing. While RhIR-activation of ant $A$ expression occurs in late stationary phase, the LasR-repression of ant $A$ occurs at the entry of stationary phase (Oglesby et al., 2008).

In this study, to better understand the growth phase-dependent QS-regulation of anthranilate in detail, we comparatively examined the QS-regulation pattern of two metabolic pathways, from anthranilate to the PQS synthesis or to TCA cycle through the growth of $P$. aeruginosa. Our data show that the two pathways are intricately regulated by the antagonistic interplay of three QS receptors at different times in growth phase mediated by two intermediate regulators, AntR and PqsR, and their cofactors.

\section{MATERIALS AND METHODS}

\section{Plasmids, bacterial strains and growth conditions}

The $P$. aeruginosa strains and plasmids used in this study are listed in Table 1. Bacteria were grown in Luria-Bertani (LB) broth containing $50 \mathrm{mM}$ MOPS [3-( $\mathrm{N}$-morpholino) propanesulphonic acid, $\mathrm{pH} 7.0$ ] at $37^{\circ} \mathrm{C}$ with vigorous shaking. Growth was monitored at $\mathrm{OD}_{600}$. Antibiotics were used at the following concentrations: ampicillin, $100 \mu \mathrm{g} / \mathrm{ml}$; tetracycline, $100 \mu \mathrm{g} / \mathrm{ml}$; gentamicin, $12.5 \mu \mathrm{g} / \mathrm{ml}$ (for E. coll) or $100 \mu \mathrm{g} / \mathrm{ml}$ (for P. aeruginosa); $\mathrm{HgCl}_{2}, 7.5 \mu \mathrm{g} / \mathrm{ml}$.

\section{RNA isolation and real-time PCR analysis}

For the real-time PCR analysis, cells from seed culture were inoculated into fresh medium (initial $\mathrm{OD}_{600}=0.01$ ) and cultivated; samples were taken at the indicated growth points for RNA extraction. The sampled cells (about $2 \times 10^{9}$ ) were directly mixed with RNA Protect Bacteria reagent (Qiagen) to stabilize RNA and lysed by lysozyme and brief sonication. RNA was purified by RNeasy mini columns (Qiagen) according to the manufacturer's protocol. Contaminated DNA was removed by on-column DNase I (Qiagen) digestion and additional RQ1 DNase I (Promega) digestion for $1 \mathrm{~h}$ at $37^{\circ} \mathrm{C}$. DNase I was removed by RNeasy column purification. We confirmed RNA integrity by agarose gel electrophoresis and ruled out genomic DNA contamination by PCR amplification with primers to a ribosomal protein gene, rplU (no amplification was detected). For the cDNA synthesis, we annealed $12 \mu \mathrm{g}$ of purified RNA with $750 \mathrm{ng}$ of semi-random decamer primers of $75 \% \mathrm{G}+\mathrm{C}$ content $\left(5^{\prime}-(\mathrm{NS})_{5}-3^{\prime}\right)$. In $30-\mu$ l reaction volume, $750 \mathrm{U}$ of Superscript II reverse transcriptase (Life Technologies), $1 \times 1$ st strand buffer, $10 \mathrm{mM}$ DTT, $0.5 \mathrm{mM}$ dNTPs, and $1 \mathrm{U}$ RNaselN (Ambion) were mixed and incubated at $25^{\circ} \mathrm{C}$ for $10 \mathrm{~min}, 37^{\circ} \mathrm{C}$ for 1 $\mathrm{h}, 42^{\circ} \mathrm{C}$ for $1 \mathrm{~h}$, and then $70^{\circ} \mathrm{C}$ for $10 \mathrm{~min}$ (for enzyme inactiva- tion). After RNA removal by alkaline hydrolysis, final cDNA products were purified by Qiaquick PCR purification kit (Qiagen) and qualified on agarose gel. For real-time PCR, primers to the genes of interest were designed using primer express software (Taqman). The $1 \mathrm{ng}$ of cDNA and $300 \mathrm{nM}$ of the specific primers were mixed in 25- $\mu$ l reaction volume of SYBR Green PCR amplification Master Mix (Applied Biosystems) in 96-well optical plate, and applied to real-time PCR machine (Applied Biosystems Model 7000). Reaction conditions were as follows: first stage, $2 \mathrm{~min}$ at $50^{\circ} \mathrm{C}$; second stage, $10 \mathrm{~min}$ at $95^{\circ} \mathrm{C}$; and third stage, 40 cycles of $15 \mathrm{~s}$ at $95^{\circ} \mathrm{C}$ (denaturation) and $1 \mathrm{~min}$ at $60^{\circ} \mathrm{C}$ (annealing and extension).

\section{Primer extension analysis}

Primer extension of pqsA was performed using the Primer Extension System (PES) AMV Reverse Transcriptase kit (Promega). The primer (5'-gatcgaaatcgaggcggaacagaa- $\left.3^{\prime}\right)$ was endlabeled by phosphorylation with $\left[\gamma \mathrm{P}^{32}\right]$-ATP, annealed with 20 $\mu \mathrm{g}$ of total RNA, and extended according to the manufacturer's protocol. The same primer was used in a sequencing reaction with Sequenase version 2.0 kit (USB). The products from the primer extension and sequencing reactions were resolved on a $6 \%$ polyacrylamide gel and visualized on X-ray film or phosphor imager (Typhoon).

\section{Construction of dual plasmid system}

To assess the activity of the regulators, we used a dual plasmid system as previously reported (Lee et al., 2006); this system is composed of the promoter-reporter fusion plasmid and the regulator-expressing plasmid. These two plasmids are compatible and replicable in both $P$. aeruginosa and $E$. coli. The transcriptional fusions, antA-lacZ (pJL201) and pqsA-lacZ (pJL301), were constructed by directional cloning of 856-bp and 631-bp PCR products of the upstream region of ant $A$ and $p q s A$ genes into the Smal site of pQF50. To express the regulators, the ant $R$ and $p q s R$ orfs were amplified using primers containing an $X$ bal site and cloned into the Xbal site of pJN105 (pJN105A and pJN105P, respectively). The correct size, orientation, and sequence of all constructs were confirmed by sequencing.

\section{$\beta$-galactosidase activity assay}

For the Pseudomonas dual plasmids assay, PAO1 was transformed with two plasmids and fresh medium was inoculated with seed culture to the initial $\mathrm{OD}_{600}=0.04$ with $0.4 \% \mathrm{~L}$ arabinose for the protein induction. Cells were collected at the indicated OD, and $\beta$-galactosidase activity was assayed by Galacto-Light Plus ${ }^{\mathrm{TM}}$ kit as recommended (Tropix). For the $E$. coli assay, $\mathrm{DH} 5 \alpha$ cells harboring two plasmids were inoculated into fresh medium to the initial $\mathrm{OD}_{600}=0.04$ and grown to $\mathrm{OD}_{600}$ $\approx 0.3$. Then, $0.4 \% \mathrm{~L}$-arabinose was added together with anthranilate or the Pseudomonas spent medium at the indicated concentration for $2 \mathrm{~h}$, and $\beta$-galactosidase activity was then assayed. Results are given in units of $\beta$-galactosidase activity per $\mathrm{OD}_{600}$. All measurements were performed at least three times and the most representative results were presented.

\section{Preparation of the Pseudomonas spent media and anthranilate assay}

A single fresh colony of each Pseudomonas strain of interest was inoculated and grown to $\mathrm{OD}_{600}=3.5$. Cells were removed by two successive centrifugations $\left(16,000 \times g\right.$ at $4^{\circ} \mathrm{C}$ for $\left.10 \mathrm{~min}\right)$ and the supernatant was taken and kept on ice for use. The spent media were always prepared freshly. To measure the anthranilate levels in the media, the spent media from the indicated OD were used in the $E$. coli dual plasmids assay with 
Table 1. Strains and plasmids used in this study

\begin{tabular}{|c|c|c|}
\hline Names & Genotype & References \\
\hline \multicolumn{3}{|l|}{$P$. aeruginosa } \\
\hline PAO1 & Wild type prototroph & Pearson et al. (1997) \\
\hline PAO-R3 & qscR mutant of PAO1, $\mathrm{Gm}^{\mathrm{R}}$ & Chugani et al. (2001) \\
\hline PAO-R1 & las $R$ mutant of $\mathrm{PAO} 1, \mathrm{Tc}^{\mathrm{R}}$ & Pearson et al. (1997) \\
\hline PDO111 & $r h I R:: \operatorname{Tn} 501, \mathrm{PAO} 1, \mathrm{Hg}^{\mathrm{R}}$ & Brint and Ohman (1995) \\
\hline PAO lasR rhIR & las $R:: T c^{R} \Delta r h I R:: \mathrm{Gm}^{R}, \mathrm{Tc}^{\mathrm{R}}, \mathrm{Gm}^{\mathrm{R}}$ & Schuster et al. (2003) \\
\hline PAO1 phnA & phnA::ISlacZ/hah (PTL 7254), Tc ${ }^{\mathrm{R}}$ & Jacobs et al. (2003) \\
\hline PAO1 phzE1 & phzE1::ISphoA/hah (PTL 37013), Tc ${ }^{\mathrm{R}}$ & Jacobs et al. (2003) \\
\hline \multicolumn{3}{|l|}{ E. coli } \\
\hline $\mathrm{DH} 5 \alpha$ & $\begin{array}{l}\text { supE44 } \phi \Delta l a c \cup 169 \text { ( } \phi 80 \text { lacZ } \Delta M 15) \text { hsdR17 } \\
\text { recA1 endA1 gyrA96 thi-1 relA1 }\end{array}$ & Sambrook et al. (1989) \\
\hline \multicolumn{3}{|l|}{ Plasmids } \\
\hline pQF50 & Broad-host-range lac $Z$ fusion vector, $A p^{R}$ & Farinha and Kropinski (1990) \\
\hline pJL201 & antA-lacZ fusion in $\mathrm{pQF50}, \mathrm{Ap}^{\mathrm{R}}$ & This work \\
\hline pJL301 & $p q s A-l a c Z$ fusion in $p Q F 50, A^{R}$ & This work \\
\hline pJN105 & araC-pBAD cassette cloned in pBBR1MCS-5 & Newman and Fuqua (1999) \\
\hline pJN105L & lasR orf in pJN105, $\mathrm{Gm}^{\mathrm{R}}$ & Lee et al. (2006) \\
\hline pJN105R & rhIR orf in pJN105, $\mathrm{Gm}^{\mathrm{R}}$ & Schuster and Greenberg (2007) \\
\hline pJN105A & antR orf in pJN105, $\mathrm{Gm}^{\mathrm{R}}$ & This work \\
\hline pJN105P & pqsR orf in pJN105, $\mathrm{Gm}^{\mathrm{R}}$ & This work \\
\hline
\end{tabular}

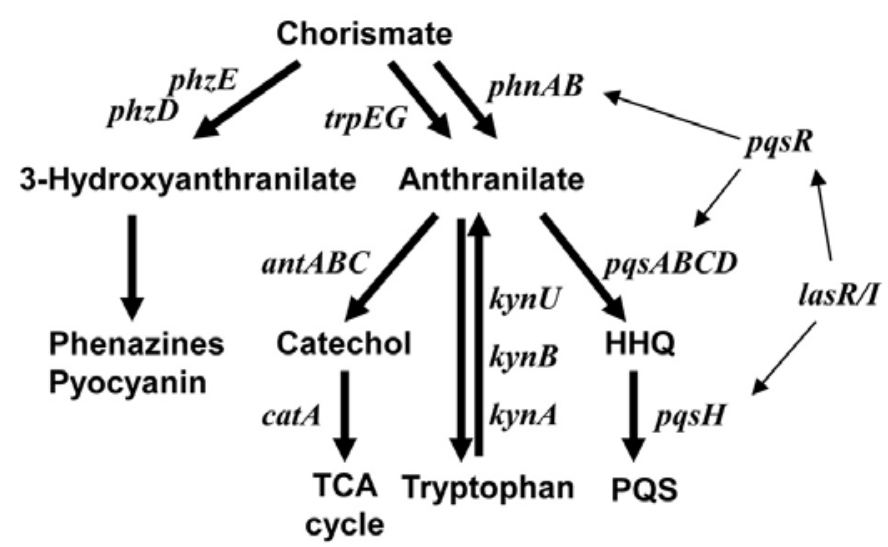

Fig. 1. Anthranilate metabolism and related genes. Arrows indicate the transcriptional activation. HHQ is 4-hydroxy-2heptylquinoline and PQS is Pseudomonas quinolone signal.

pJN105A and pJL201, and $\beta$-galactosidase activity was assayed as described above.

\section{RESULTS}

\section{Metabolic pathways from anthranilate are growth phase- differentially regulated by the antagonistic interplay of three QS regulators}

To address details of the timing and antagonistic regulation of anthranilate metabolism by QS systems, we investigated whether the expression of $c a t A$, a gene at the next step in the anthranilate degradation pathway, is synchronously regulated with ant $A$. The real-time PCR analysis showed that cat $A$ is regulated in a pattern similar to $a n t A$, where the cat $A$ expression was induced in late stationary phase, which disappeared in $r h I R$ mutant, and dramatically derepressed in $q s c R$ mutant (Fig. 2). Transcriptome analysis also showed RhIR-dependent activation and LasR-dependent repression of catA (Schuster et al., 2003). Together with the ant $A$ regulation pattern, this indicated that LasR could repress the expression of anthranilate degradation function during exponential and early stationary phases, but the C4-activated RhIR could overcome this repression in the late stationary phase to activate anthranilate degradation.
For better comparison, we investigated the expression timing and patterns of the pqsABCDE/phnAB gene cluster in another pathway toward the PQS synthesis by real-time PCR analysis under the same conditions. Because pqsABCDE and phnAB are synchronously regulated by the QS system (Deziel et al., 2004; Schuster et al., 2003), we probed the phnA expression. Our results showed that the pqsA-E/phnAB gene cluster was derepressed in the $r h I R$ mutant throughout growth, and while it was activated at late log phase in wild type, its activation was delayed to the late stationary phase in the las $R$ mutant (Fig. 2). This indicated that LasR affected the timing of pqsA-E/phnAB gene cluster induction but not the expression level. Instead, the expression level of this gene cluster was more strongly regulated by RhIR, as demonstrated by the much higher level of expression in $r h / R$ mutant (Fig. 2). Like the antA and catA expressions, the most dramatic change in the expression of the $p q s A-E / p h n A B$ gene cluster was observed in the $q s c R$ mutant, where both the expression level and timing were particularly altered. The expression of $p q s A-E / p h n A B$ gene cluster was much advanced toward early exponential phase and also derepressed to a high level (Fig. 2).

While pqsA-E/phnAB genes in a cluster have been reported to be synchronously regulated by QS (Schuster et al., 2003), an 


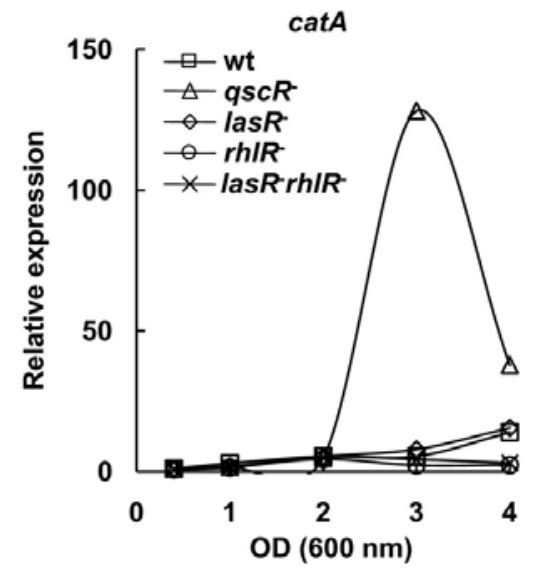

Fig. 2. The expression patterns of $\operatorname{cat} A$ and $p h n A$. The left panel shows the expression patterns of catA, and the right panel, that of $p h n A$ in various QS mutants throughout growth, as measured by real-time PCR analysis. The expression levels are indicated as relative expression units when the expression level of wild-type $\mathrm{PAO} 1$ at $\mathrm{OD}_{600}=0.4$ is 1 . independent study claimed that pqs $A-E$ and $p h n A B$ genes are separately transcribed as two distinct polycistronic operons (McGrath et al., 2004). To exclude any possibility that the expression pattern of $p h n A$ might be different from that of $p q s A$, we confirmed the pqsA expression by primer extension analysis, finding that the pqs $A$ expression pattern was same as that of phnA in Fig. 2 (Fig. 3). Therefore, these particular expression patterns of the pqsA-E/phnAB gene cluster in $r h I R$ and $q s c R$ mutants are most likely achieved by transcriptional regulation on the pqsA promoter.

Interestingly, the RhIR repression affected the expression of the pqsA-E/phnAB gene cluster even in the early exponential phase when RhIR is not fully active. Because the $r h l$ system is dependent on the las system in the QS hierarchy (Latifi et al., 1996; Pesci et al., 1997), this early repression of the LasRactivated gene by RhIR was not expected. Another interesting regulation was found in the las $R$ rhlR double mutant where the derepression of the pqsA-E/phnAB gene cluster was detected only in late stationary phase (Fig. 2). This implied that other factor(s) may be involved in the activation of this operon in late stationary phase, which is repressed by RhIR in wild type.

These results showed the growth phase-differential regulation of anthranilate metabolism by the antagonistic interplay of three QS regulators, LasR, RhIR, and QscR, where the destination of anthranilate is finely tuned by QS systems depending on the growth phase.

Two direct regulators, AntR and PqsR, mediate this welltimed QS regulation, partly through the QS-regulated expression of ant $R$ and $p q s R$ genes

For PQS synthesis, PqsR has been reported as a direct activator of pqsA promoter (McGrath et al., 2004; Wade et al., 2005). For the antABC operon, AntR (encoded by PA2511), which is divergently transcribed from ant $A$, has recently been suggested as a direct activator (Oglesby et al., 2008). Therefore, the QS regulation of anthranilate degradation and PQS synthesis could be indirectly achieved through the regulation of ant $R$ and $p q s R$ expression. However, the recently reported expression patterns and timings of ant $R$ and $p q s R$ were different from those of an$t A B C /$ cat $A$ and $p q s A-E / p h n A B$ operons. As shown in Fig. 2, the pqsA-E/phnAB gene cluster was differently expressed in $r h / R$ and $q s c R$ mutants (Fig. 2), whereas pqsR was similarly expressed in both mutants (Oglesby et al., 2008). This means that the pqs $R$ expression pattern does not fully reflect the $p q s A$ expression pattern. Moreover, when we modified the expression level and timing of LasR and RhIR by arabinose-induced overexpression in PAO1 cells, the well-timed regulation was not

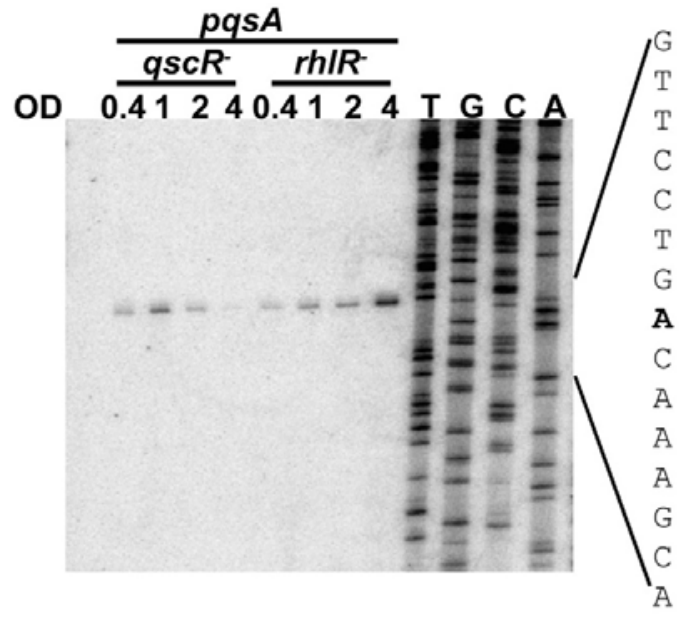

CCCCGAAACTTTTTCGTTCGGACTCCGAATATCGCGC

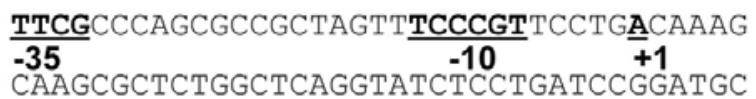

\section{AtAtCGCTgAagaggaAagtTCtgtCATg > PqsA}

Fig. 3. Primer extension analysis on $p q s A$ promoter. Primer extension experiments were quantitatively carried out to measure the transcript level of the pqs $A$ gene. The information about the $p q s A$ promoter such as +1 sites, $-35 /-10$ boxes, and the start codon was indicated by underlines and bold letters. RNAs were extracted from the indicated growth points $\left(\mathrm{OD}_{600}\right)$ of each mutant strain.

significantly altered (Fig. 4). So, it was apparent that the complex regulation of anthranilate metabolism requires something other than the QS regulation of the antR and pqs $R$ gene expressions.

Notably, we found PqsR repressed antA expression (Fig. 4) as recently reported (Oglesby et al., 2008). This indicates that PqsR can drive PQS production both by activating the PQS synthetic genes and by repressing the anthranilate degrading genes at the same time.

\section{Cofactors of AntR and PqsR are QS-dependently} produced

Since AntR and PqsR require cofactors, anthranilate and PQS 
A

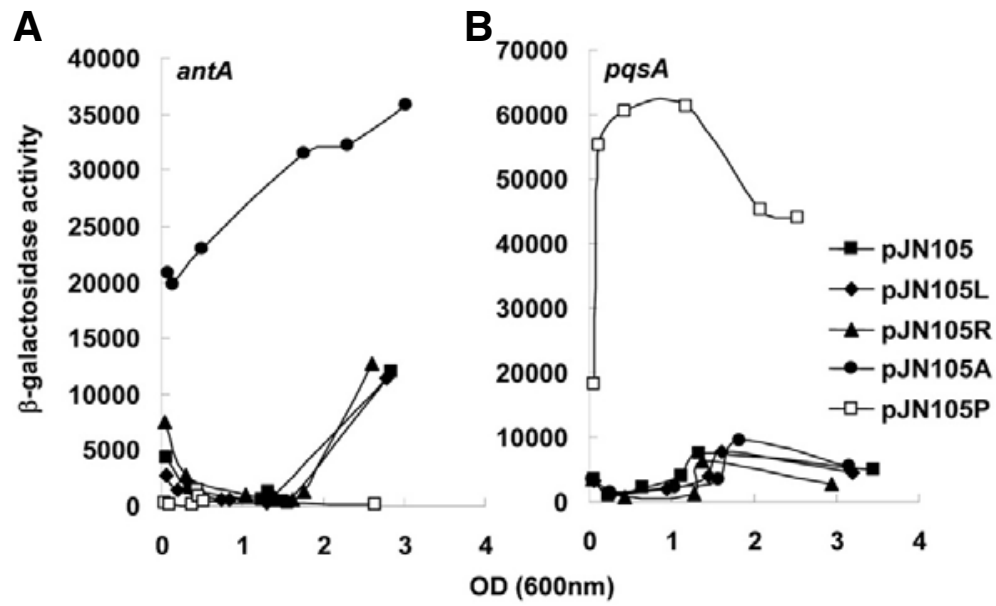

A
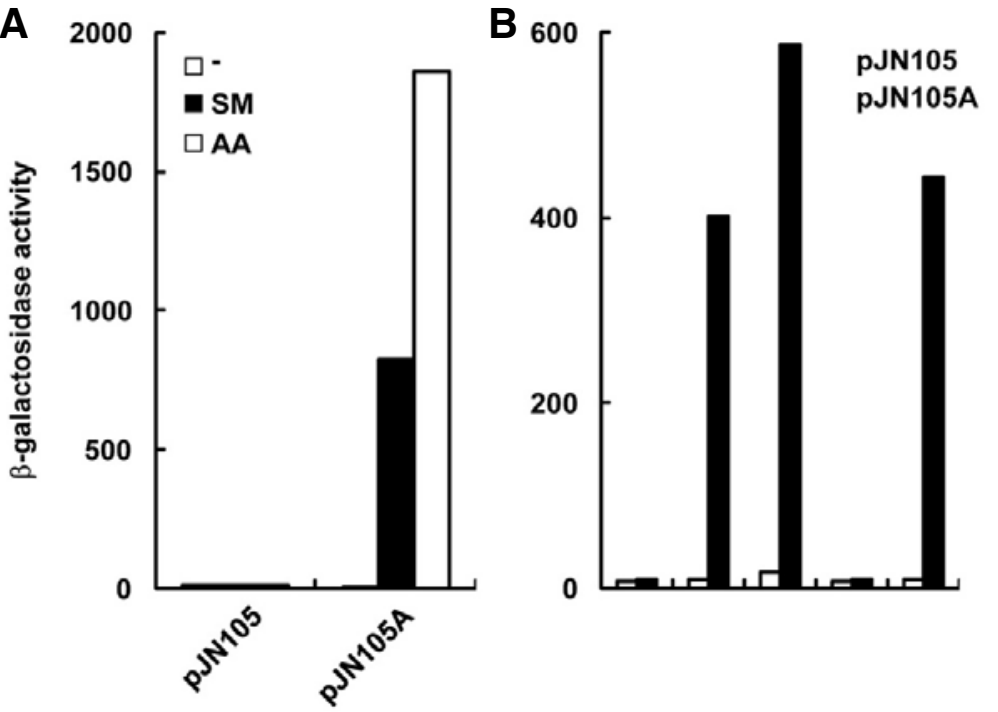

Fig. 4. Effect of the overexpression of AntR, PqsR, and the QS regulators on the expression of $\operatorname{ant} A(\mathrm{~A})$ and $p q s A(B)$ in $P$. aeruginosa $P A O 1$. The regulatorexpressing plasmid (pJN105P, pJN105A, pJN105L, or pJN105R) was transformed into PAO1 cells that carried reporter fusion plasmid (pJL201 or pJL301). Cells containing two plasmids were grown with $0.4 \%$ L-arabinose, and $\beta$-galactosi-dase activity was measured at the indicated growth points throughout growth.

Fig. 5. Anthranilate-dependent activation of antA (A) and RhIR-dependent secretion of anthranilate. (A) the secreted anthranilate in the Pseudomonas spent medium was detected using the $E$. coli dual plasmid assay, which has two compatible plasmids, pJN105A and pJL201 (antA-lacZ). Cells were treated with 0.1 $\mathrm{mM}$ anthranilate (AA) or $20 \%$ PAO1 spent medium (SM) for $3 \mathrm{~h}$. (B) treatment with the Pseudomonas spent medium from various $\mathrm{R}$ mutants at $20 \%$ for $3 \mathrm{~h}$ and $\beta$-gal activity was measured.

(or HHQ), respectively (Oglesby et al., 2008; Wade et al., 2005; Xiao et al., 2006), cofactor production could affect the QS regulation of anthranilate metabolism. The PQS and HHQ production is known to be activated by the las system (McGrath et al., 2004). However, which QS system influences the anthranilate production responsible for AntR activation was not clear. phnAB encodes the anthranilate synthase that is responsible for PQS production, but as shown in Fig. 2, the phnAB expression is facilitated by LasR and repressed by RhIR, which is opposite to the RhIR-dependent expression patterns of antA and cat $A$. Another important anthranilate source for PQS synthesis is the kynurenine pathway, in which anthranilate is supplied through tryptophan degradation (Farrow and Pesci, 2007; Oglesby et al., 2008). However, the genes involved in this pathway, kynB and $k y n U$, were positively regulated by las $R$ function (Schuster et al., 2003). This implied that the anthranilate produced by phnAB or kynurenine pathways might be insufficient or not responsible for the activation of the AntR function. So, we investigated the excretion of anthranilate in the spent media from various QS mutants, using an $E$. coli dual plasmid reporter system. This system carries AntR-expressing and ant $A$-lac $Z$ fusion plasmids that sensitively respond to anthranilate in the spent medium (Fig. 5A). Of the spent media from various QS mutants, only one from the rhIR mutant failed to activate AntR (Fig. 5B).
Since anthranilate is considered permeable and the exogenous supplement of anthranilate can complement intracellular function (Farrow and Pesci, 2007), this result indicated that the anthranilate accumulation is RhIR-dependent. As the antA and cat $A$ expressions were turned on in late stationary phase (Fig. 2) (Oglesby et al., 2008), the anthranilate excretion also occurred in late stationary phase (Fig. 6). These results suggested that anthranilate might accumulate at late stationary phase to a sufficient level to activate AntR and this accumulation is RhIRdependent. Consistently, the exogenous addition of anthranilate to a level high enough to activate AntR advanced the antA expression (Fig. 7).

As mentioned above, this increase of anthranilate is not likely dependent on either phnAB or kynurenine pathways, and is phnA-independent (Fig. 6). This means that anthranilate should accumulate due to another factor that is activated by RhIR. There are multiple anthranilate synthase homologues in addition to PhnAB in $P$. aeruginosa (Essar et al., 1990; Farrow and Pesci, 2007; Gallagher et al., 2002; Stover et al., 2000). Two of them, PhzE1 and PhzE2 (responsible for phenazine synthesis), are activated in an RhIR-dependent manner, whereas others such as PabB (responsible for para-aminobenzoate synthesis), PchA (responsible for salicylate synthesis) and TrpEG (responsible for tryptophan synthesis) are not QS-dependent (Schuster 


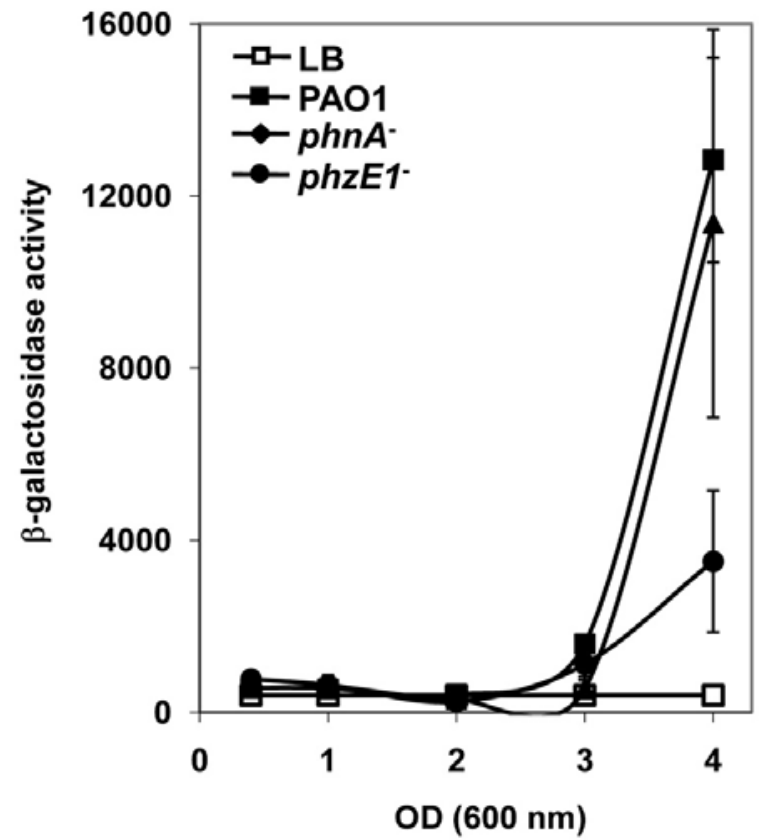

Fig. 6. The phnAB-independent secretion of anthranilate in late stationary phase. The anthranilate in the spent media from phnAand phzE1 mutant strains were detected throughout growth by using the $E$. coli dual plasmid assay used in Fig. 5. Since the $\beta$ galactosidase activity of this bioassay has fairly good linear correlation with the concentration of exogenously added synthetic anthranilate, this method was used to estimate the anthranilate level. The anthranilate level in the culture media of wild-type PAO1 at late stationary phase $\left(\mathrm{OD}_{600}=4\right)$ was estimated at $49.3 \mu \mathrm{M}$ and that of phzE1 mutant was at $13.5 \mu \mathrm{M}$.

\section{et al., 2003).}

PhzE1 and PhzE2 have almost identical amino acid sequences, and the PhzEs have been suggested as an anthranilate synthase lacking the lyase activity of TrpE subunits, which cleaves pyruvate from 2-amino-deoxychorismic acid (ADC) (Mavrodi et al., 2006). So, the product of the PhzE-catalyzed reaction has been known to be $A D C$, an anthranilate analogue that has a pyruvate moiety. Together with PhzD, PhzE has been suggested to generate 3-hydroxyanthranilate instead of anthranilate (Fig. 1).

The expression patterns of phzE1 and 2 are similar, but the expression level of phzE1 has been known to be much higher than that of phzE2 (Ledgham et al., 2003; Schuster et al., 2003). We suspected that the phzE might be responsible for the RhIRdependent anthranilate accumulation, because both phz operons are RhIR-dependently expressed in stationary phase (Chugani et al., 2001; Ledgham et al., 2003; Schuster et al., 2003), and their expressions are derepressed and advanced in qscR mutant (Chugani et al., 2001; Ledgham et al., 2003; Lequette et al., 2006), which is similar with the ant $A$ and cat $A$ expression patterns. As we expected, the anthranilate level in the culture medium of the phzE1 mutant was lower than those in wild type (Fig. 6). To exclude the possibility that our AntR reporter system nonspecifically detected 3-hydroxyanthranilate, we tested the synthetic 3-hydroxyanthranilate for AntR activation and found that 3-hydroxyanthranilate failed to activate AntR significantly (Fig. 8). This suggests that anthranilate is accumulated in late stationary phase by $r h$ l function and the anthrani-

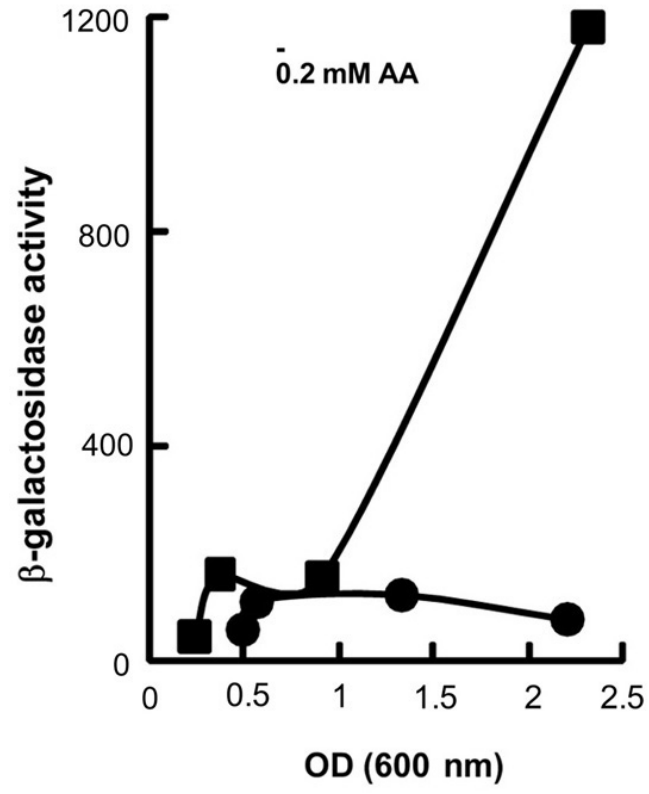

Fig. 7. Advanced activation of ant $A$ by the exogenously added anthranilate in $P$. aeruginosa $\mathrm{PAO} 1$. The antA-lac $Z$ fusion plasmid (pJL201) was transformed into $P$. aeruginosa PAO1 and cultivated in LB broth containing $0.2 \mathrm{mM}$ anthranilic acid (AA). Aliquots of culture were taken and $\beta$-galactosidase activity was assayed.

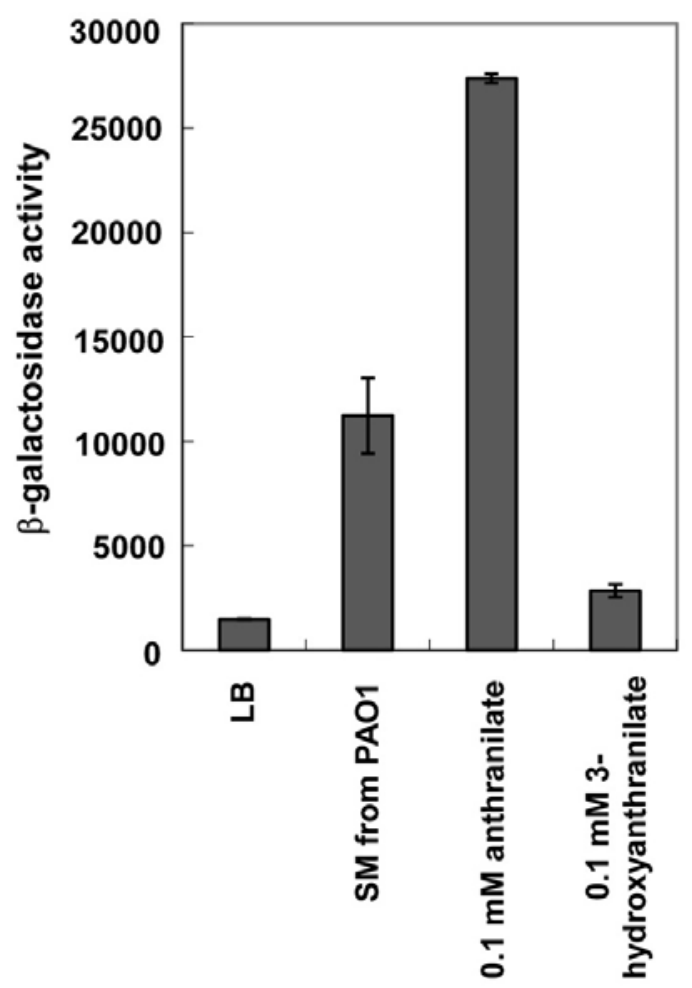

Fig. 8. Specific response of AntR to anthranilate. E. coli dual plasmid assay used in Fig. 5 was treated with anthranilate and 3-hydroxyanthranilate, and $\beta$-galactosidase activity was assayed. Spent medium (SM) from PAO1 overnight culture was added to $20 \%$ and fresh LB medium was used as control. 


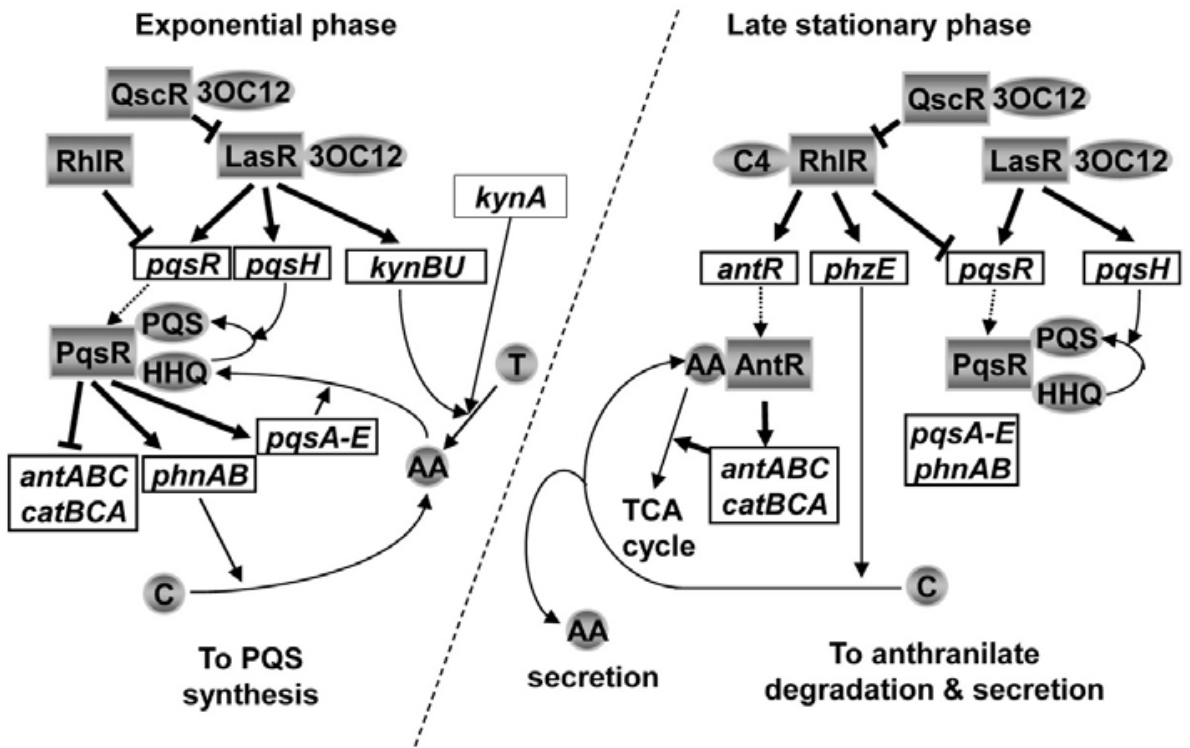

\section{AA anthranilate C chorismate $\mathrm{T}$ tryptophan HHQ 4-hydroxy-2-heptylquinoline}

Fig. 9. Summary of the proposed regulatory circuit. The anthranilate metabolism is differentially regulated along with the growth phase. Until onset of stationary phase (left), 3OC12-activated LasR activates the PQS synthesis by increasing the PqsR level (Deziel et al., 2004; Oglesby et al., 2008; Schuster et al., 2003; Wade et al., 2005). PqsR also represses anthranilate degradation functions (Fig. 4). During this period, anthranilate as PQS precursor can be supplied from chrismate and tryptophan by the functions of phnAB and kynA $B U$ (Calfee et al., 2001; Farrow and Pesci, 2007; Gallagher et al., 2002), which are activated by LasR (Fig. 2) (Schuster et al., 2003), and RhIR represses the PQS synthesis (Figs. 2 and 3). Even though RhIR is supposed to be inactive at early exponential phase because of the premature C4-production, RhIR is likely to repress the pqsR function (Figs. 2 and 3) (Oglesby et al., 2008). In stationary phase (right), the C4activated RhIR function inhibits PqsR (Fig. 2), which may remove the repression on the antA expression and interrupt the conversion of anthranilate to PQS. The RhIR-activated antR expression and anthranilate accumulation turn on the anthranilate degradation (Figs. 2, 5, and 6) (Oglesby et al., 2008), and the RhIR-activated phzE might influence the anthranilate excretion and accumulation (Fig. 6). QscR strongly represses both las $R$ and $r / R$ functions, but in a growth phase-differential manner (Figs. 2 and 3 ). See the text for details. The genes are represented in white boxes, proteins in gray boxes, small molecules in gray circles.

late accumulation may be significantly influenced by the $\mathrm{rhl}$ dependent $p h z E$ function, although it has been previously suggested to be responsible for phenazine synthesis.

\section{DISCUSSION}

Together with published data and suggestions, our results can be incorporated into the proposed regulatory circuit as summarized in Fig. 9. In this study, we showed that the induction and repression of genes in anthranilate metabolism are tuned along with growth by the antagonistic interplay among three QS regulators, LasR, RhIR, and QscR, although there is no direct regulation by these regulators. Even QscR, which showed the most dramatic repression of the expressions of antA and pqs $A$ in vivo, did not bind to any promoter of these genes in vitro (Lee et al., 2006). Apparently QS systems affect all regulations through the direct regulators, AntR and PqsR. As it has been suggested that an unknown 'super-regulation' influences QS and the induction of most target genes is not simply affected by levels of signals or receptor proteins in $P$. aeruginosa (Schuster and Greenberg, 2007; Schuster et al., 2003), our results also show the complicated QS regulation around anthranilate metabolism, such that the overexpression of LasR or RhIR failed to disrupt this well-timed regulation (Fig. 3). This means that the activities of AntR and PqsR could not be immediately altered by changing the level of QS regulators, and some other factors should be involved in the 'super-regulation'.

One reason for this complicated regulation could be the fine QS-regulation in the production of cofactors for AntR and PqsR. This means that QS systems regulate anthranilate metabolism both through the expressions of antR and pqs $R$ genes and through cofactor production. While PhnAB has been suggested as the anthranilate synthase responsible for PQS synthesis
(Calfee et al., 2001; Gallagher et al., 2002), many publications claimed the presence of multiple synthases and sources of anthranilate (Aendekerk et al., 2005; Essar et al., 1990; Farrow and Pesci, 2007; Gallagher et al., 2002). Our result also suggested that another possible factor influenced anthranilate accumulation in late stationary phase. While the other anthranilate sources, such as the phnAB function, kynurenine pathway, or trpEG are LasR-dependent or QS-independent (Schuster et al., 2003), the anthranilate excretion was RhIR-dependent (Fig. 5). This apparent inconsistency led us to investigate another anthranilate synthase, PhzE, which is rhIR-dependently induced in stationary phase (Schuster et al., 2003) and strongly derepressed and advanced in qscR mutant (Chugani et al., 2001; Ledgham et al., 2003; Lequette et al., 2006). Our result showed reduced excretion of anthranilate in phzE1 mutant (Fig. 6). We assume that the anthranilate produced by PhnAB or tryptophan degradation until stationary phase might be rapidly consumed in PQS synthesis by PqsA-D and thus does not accumulate, but once RhIR is activated, the RhIR-induced PhzE could influence the anthranilate accumulation in stationary phase. However, although PhzE is homologous to other anthranilate synthases, its function has been postulated to produce 3-hydroxyanthranilate instead of anthranilate (Mavrodi et al., 2001). But since 3-hydroxyanthranilate did not activate AntR significantly (Fig. 8), it is still unknown how PhzE affects anthranilate accumulation.

As shown in Fig. 2, pqsA was constantly up-regulated throughout the growth in the $r h / R$ mutant, whereas its expression in $q s c R$ mutant was activated in a much earlier phase and rapidly decreased in stationary phase. Interestingly, the pqs $R$ expression pattern was similar in both mutants but the level was higher in the mutants than in wild type at early log phase (Oglesby et al., 2008). Although the repressions of pqsR by 
RhIR and QscR can explain the higher expression of pqs $A$ in $q s c R$ and $r h I R$ mutants at early log phase, it is hard to explain why the pqs $A$ expression in late stationary phase was decreased in the $q s c R$ mutant but continuously increased in the $r h I R$ mutant. This result strongly suggests an inhibitory effect on PqsR activity at the post-transcriptional level, which may be activated by RhIR but repressed by QscR in stationary phase.

Interestingly, QscR acted as a repressor of both antA/catA and $p q s A-E / p h n A B$ operons in a growth phase-differential manner, where the qscR mutation strongly derepressed $p q s A$ $E / p h n A B$ during log phase, but repressed it in stationary phase; in contrast, the same $q s c R$ mutation strongly derepressed ant $A$ cat $A$ only in late stationary phase (Fig. 2). The log phasederepression of $p q s A-E / p h n A B$ by the $q s c R$ mutation may be due to the higher expression level of $p q s R$ in the $q s c R$ mutant and self-boosting effect by higher expression of pqsA-E/phnAB in the $q s c R$ mutant. One clue for the stationary phase-repression of pqsA-E/phnAB by the qscR mutation may be the anthranilate accumulation, in that the anthranilate accumulation can be facilitated by the $q s c R$ mutation because QscR strongly represses both phzE1 and 2 (Chugani et al., 2001; Ledgham et al., 2003; Lequette et al., 2006). The opposite attitude toward phzE expression may be a reason why RhIR and QscR show a similar repressive effect on pqsA-E/phnAB during log phase, but a dramatically different effect in stationary phase.

\section{ACKNOWLEDGMENTS}

This work was supported by the Korea Research Foundation Grant funded by the Korean Government (KRF-2007-331C00222), the Korea Science and Engineering Foundation (KOSEF) grant funded by the Korea government Ministry of Science and Technology (No. R01-2007-000-20732-0), and Korea Ministry of Environment as "The Eco-technopia 21 project" (No. 102-081-067). This work was also supported by Basic Science Research Program through the National Research Foundation of Korea (NRF) funded by the Ministry of Education, Science and Technology (2010-0006622) and National Research Foundation of Korea Grant funded by the Korean Government (2010-0015901).

\section{REFERENCES}

Aendekerk, S., Diggle, S.P., Song, Z., Hoiby, N., Cornelis, P., Williams, P., and Camara, M. (2005). The MexGHI-OpmD multidrug efflux pump controls growth, antibiotic susceptibility and virulence in Pseudomonas aeruginosa via 4-quinolone-dependent cell-to-cell communication. Microbiology 151, 1113-1125.

Brint, J.M., and Ohman, D.E. (1995). Synthesis of multiple exoproducts in Pseudomonas aeruginosa is under the control of RhIRRhll, another set of regulators in strain PAO1 with homology to the autoinducer-responsive LuxR-Luxl family. J. Bacteriol. 177, 7155-7163.

Calfee, M.W., Coleman, J.P., and Pesci, E.C. (2001). Interference with Pseudomonas quinolone signal synthesis inhibits virulence factor expression by Pseudomonas aeruginosa. Proc. Natl. Acad. Sci. USA 98, 11633-11637.

Chugani, S.A., Whiteley, M., Lee, K.M., D’Argenio, D., Manoil, C., and Greenberg, E.P. (2001). QscR, a modulator of quorumsensing signal synthesis and virulence in Pseudomonas aeruginosa. Proc. Natl. Acad. Sci. USA 98, 2752-2757.

Deziel, E., Lepine, F., Milot, S., He, J., Mindrinos, M.N., Tompkins, R.G., and Rahme, L.G. (2004). Analysis of Pseudomonas aeruginosa 4-hydroxy-2-alkylquinolines (HAQs) reveals a role for 4hydroxy-2-heptylquinoline in cell-to-cell communication. Proc. Natl. Acad. Sci. USA 101, 1339-1344.

Diggle, S.P., Winzer, K., Chhabra, S.R., Worrall, K.E., Camara, M., and Williams, P. (2003). The Pseudomonas aeruginosa quinolone signal molecule overcomes the cell density-dependency of the quorum sensing hierarchy, regulates $r$ hl-dependent genes at the onset of stationary phase and can be produced in the ab- sence of LasR. Mol. Microbiol. 50, 29-43.

Essar, D.W., Eberly, L., Hadero, A., and Crawford, I.P. (1990). Identification and characterization of genes for a second anthranilate synthase in Pseudomonas aeruginosa: interchangeability of the two anthranilate synthases and evolutionary implications. J. Bacteriol. 172, 884-900.

Farinha, M.A., and Kropinski, A.M. (1990). Construction of broadhost-range plasmid vectors for easy visible selection and analysis of promoters. J. Bacteriol. 172, 3496-3499.

Farrow, J.M., 3rd, and Pesci, E.C. (2007). Two distinct pathways supply anthranilate as a precursor of the Pseudomonas quinolone signal. J. Bacteriol. 189, 3425-3433.

Fuqua, C., Parsek, M.R., and Greenberg, E.P. (2001). Regulation of gene expression by cell-to-cell communication: acyl-homoserine lactone quorum sensing. Annu. Rev. Genet. 35, 439-468.

Gallagher, L.A., McKnight, S.L., Kuznetsova, M.S., Pesci, E.C., and Manoil, C. (2002). Functions required for extracellular quinolone signaling by Pseudomonas aeruginosa. J. Bacteriol. 184, 64726380 .

Jacobs, M.A., Alwood, A., Thaipisuttikul, I., Spencer, D., Haugen, E., Ernst, S., Will, O., Kaul, R., Raymond, C., Levy, R., et al. (2003). Comprehensive transposon mutant library of Pseudomonas aeruginosa. Proc. Natl. Acad. Sci. USA 100, 14339-14344.

Kim, C., Kim, J., Park, H.Y., Park, H.J., Kim, C.K., Yoon, J., and Lee, J.H. (2009). Development of inhibitors against TraR quorumsensing system in Agrobacterium tumefaciens by molecular modeling of the ligand-receptor interaction. Mol. Cells 28 , 447453.

Latifi, A., Foglino, M., Tanaka, K., Williams, P., and Lazdunski, A. (1996). A hierarchical quorum-sensing cascade in Pseudomonas aeruginosa links the transcriptional activators LasR and RhIR (VsmR) to expression of the stationary-phase sigma factor RpoS. Mol. Microbiol. 21, 1137-1146.

Ledgham, F., Ventre, I., Soscia, C., Foglino, M., Sturgis, J.N., and Lazdunski, A. (2003). Interactions of the quorum sensing regulator QscR: interaction with itself and the other regulators of Pseudomonas aeruginosa LasR and RhIR. Mol. Microbiol. 48, 199210.

Lee, J.H., Lequette, Y., and Greenberg, E.P. (2006). Activity of purified QscR, a Pseudomonas aeruginosa orphan quorum-sensing transcription factor. Mol. Microbiol. 59, 602-609.

Lequette, Y., Lee, J.H., Ledgham, F., Lazdunski, A., and Greenberg, E.P. (2006). A distinct QscR regulon in the Pseudomonas aeruginosa quorum-sensing circuit. J. Bacteriol. 188, 3365-3370.

Mavrodi, D.V., Bonsall, R.F., Delaney, S.M., Soule, M.J., Phillips, G., and Thomashow, L.S. (2001). Functional analysis of genes for biosynthesis of pyocyanin and phenazine-1-carboxamide from Pseudomonas aeruginosa PAO1. J. Bacteriol. 183, 6454-6465.

Mavrodi, D.V., Blankenfeldt, W., and Thomashow, L.S. (2006). Phenazine compounds in fluorescent Pseudomonas spp. biosynthesis and regulation. Annu. Rev. Phytopathol. 44, 417-445.

McGrath, S., Wade, D.S., and Pesci, E.C. (2004). Dueling quorum sensing systems in Pseudomonas aeruginosa control the production of the Pseudomonas quinolone signal (PQS). FEMS Microbiol. Lett. 230, 27-34.

McKnight, S.L., Iglewski, B.H., and Pesci, E.C. (2000). The Pseudomonas quinolone signal regulates rhl quorum sensing in Pseudomonas aeruginosa. J. Bacteriol. 182, 2702-2708.

Newman, J.R., and Fuqua, C. (1999). Broad-host-range expression vectors that carry the L-arabinose-inducible Escherichia coli araBAD promoter and the araC regulator. Gene 227, 197-203.

Oglesby, A.G., Farrow, J.M., 3rd, Lee, J.H., Tomaras, A.P., Greenberg, E.P., Pesci, E.C., and Vasil, M.L. (2008). The influence of iron on Pseudomonas aeruginosa physiology: a regulatory link between iron and quorum sensing. J. Biol. Chem. 283, 1555815567.

Pearson, J.P., Pesci, E.C., and Iglewski, B.H. (1997). Roles of Pseudomonas aeruginosa las and $r h l$ quorum-sensing systems in control of elastase and rhamnolipid biosynthesis genes. J. Bacteriol. 179, 5756-5767.

Pesci, E.C., Pearson, J.P., Seed, P.C., and Iglewski, B.H. (1997). Regulation of las and rhl quorum sensing in Pseudomonas aeruginosa. J. Bacteriol. 179, 3127-3132.

Pesci, E.C., Milbank, J.B., Pearson, J.P., McKnight, S., Kende, A.S., Greenberg, E.P., and Iglewski, B.H. (1999). Quinolone signaling in the cell-to-cell communication system of Pseudomonas aeruginosa. Proc. Natl. Acad. Sci. USA 96, 11229-11234. 
Sambrook, J.E., Fritsch, F., and Maniatis, T. (1989). Molecular cloning: a laboratory manual. (Cold Spring Harbor, N.Y., USA: Cold Spring Harbor Laboratory Press).

Schuster, M., and Greenberg, E.P. (2006). A network of networks: quorum-sensing gene regulation in Pseudomonas aeruginosa. Int. J. Med. Microbiol. 296, 73-81.

Schuster, M., and Greenberg, E.P. (2007). Early activation of quorum sensing in Pseudomonas aeruginosa reveals the architecture of a complex regulon. BMC Genomics 8, 287.

Schuster, M., Lostroh, C.P., Ogi, T., and Greenberg, E.P. (2003). Identification, timing, and signal specificity of Pseudomonas aeruginosa quorum-controlled genes: a transcriptome analysis. J. Bacteriol. 185, 2066-2079.

Stover, C.K., Pham, X.Q., Erwin, A.L., Mizoguchi, S.D., Warrener, P., Hickey, M.J., Brinkman, F.S., Hufnagle, W.O., Kowalik, D.J., Lagrou, M., et al. (2000). Complete genome sequence of Pseu- domonas aeruginosa PA01, an opportunistic pathogen. Nature 406, 959-964.

Van Delden, C., and Iglewski, B.H. (1998). Cell-to-cell signaling and Pseudomonas aeruginosa infections. Emerg. Infect. Dis. 4, 551560.

Venturi, V. (2006). Regulation of quorum sensing in Pseudomonas. FEMS Microbiol. Rev. 30, 274-291.

Wade, D.S., Calfee, M.W., Rocha, E.R., Ling, E.A., Engstrom, E., Coleman, J.P., and Pesci, E.C. (2005). Regulation of Pseudomonas quinolone signal synthesis in Pseudomonas aeruginosa. J. Bacteriol. 187, 4372-4380.

Xiao, G., Deziel, E., He, J., Lepine, F., Lesic, B., Castonguay, M.H., Milot, S., Tampakaki, A.P., Stachel, S.E., and Rahme, L.G. (2006). MvfR, a key Pseudomonas aeruginosa pathogenicity LTTR-class regulatory protein, has dual ligands. Mol. Microbiol. 62, 1689-1699. 
